\title{
Estimation of effective doses in pediatric X-ray computed tomography examination
}

\author{
HIDEKI OBARA $^{1,2}$, MIDORI TAKAHASHI $^{1}$, KAZUYA KUDOU $^{1}$, YASUSHI MARIYA ${ }^{1}$, \\ YOSHIHIRO TAKAI ${ }^{3}$ and IKUO KASHIWAKURA ${ }^{1}$
}

\author{
${ }^{1}$ Department of Radiological Life Sciences, Division of Medical Life Sciences, Hirosaki University Graduate School of \\ Health Sciences, Hirosaki, Aomori 036-8564; ${ }^{2}$ Department of Radiology, Division of Medical Technology, \\ Hirosaki University School of Medicine and Hospital, Hirosaki, Aomori 036-8563; ${ }^{3}$ Department of Radiology \\ and Radiation Oncology, Hirosaki University School of Medicine, Hirosaki, Aomori 036-8562, Japan
}

Received May 19, 2016; Accepted March 23, 2017

DOI: $10.3892 / e t m .2017 .5102$

\begin{abstract}
X-ray computed tomography (CT) images are used for diagnostic and therapeutic purposes in various medical disciplines. In Japan, the number of facilities that own diagnostic $\mathrm{CT}$ equipment, the number of $\mathrm{CT}$ examinations and the number of CT scanners increased by $\sim 1.4$-fold between 2005 and 2011. CT operators (medical radiological technologists, medical physicists and physicians) must understand the effective doses for examinations at their own institutions and carefully approach each examination. In addition, the patients undergoing the examination (as well as his/her family) must understand the effective dose of each examination in the context of the cumulative dose. In the present study, the numbers of pediatric patients (aged 0-5 years) and total patients who underwent CT at Hirosaki University Hospital (Hirosaki, Japan) between January 2011 and December 2013 were surveyed, and effective doses administered to children aged 0,1 and 5 years were evaluated. Age- and region-specific conversion factors and dose-length products obtained from the CT scanner were used to estimate the effective doses. The numbers of CT examinations performed in 2011, 2012 and 2013 were 16,662, 17,491 and 17,649, respectively, of which $613(1.2 \%)$ of the overall total involved children aged $0-5$ years. The estimated effective doses per examination to children aged 0,1 and 5 years were $6.3 \pm 4.8,4.9 \pm 3.8$ and $2.7 \pm 3.0 \mathrm{mSv}$, respectively. This large variation was attributed to several factors associated with scan methods and ranges in actual setting. In conclusion, the requirement for individual patient prospective exposure management systems and estimations of
\end{abstract}

Correspondence to: Professor Ikuo Kashiwakura, Department of Radiological Life Sciences, Division of Medical Life Sciences, Hirosaki University Graduate School of Health Sciences, 66-1 Hon, Hirosaki, Aomori 036-8564, Japan

E-mail: ikashi@hirosaki-u.ac.jp

Key words: computed tomography, effective dose, dose-length product conversion factors low-dose radiation exposure should be considered in light of the harmful effects of exposure.

\section{Introduction}

X-ray computed tomography (CT) is a medical imaging technique in which computer-processed $\mathrm{X}$-ray projections are used to produce tomographic images or slices of specific areas of the body. Since 2000, the importance of multi-detector CT, which permits faster scanning and a wider range of clinical applications, has been recognized (1). According to a report from the Ministry of Health, Labour and Welfare of Japan, the number of facilities that owned diagnostic CT equipment, the number of CT examinations and the number of CT scanners in 2005 were 8,149 [1-month (September) total], 1,634,056 [1-month (September) total] and 8,903 (as of October 1st), respectively (2). By 2011, these numbers had increased to 11,415 [1-month (September) total], 2,357,580 [1-month (September) total] and 12,482 (as of October 1st), respectively (3), demonstrating a $\sim 1.4$-fold increase relative to 2005 .

A study by Tsushima et al (4) indicated that CT is accountable for more than half of the radiation exposure incurred from diagnostic imaging. The authors noted that unfortunately no reliable data regarding radiation exposure from radiological imaging are available; it is expected that the situation in Japan is similar. The International Commission on Radiological Protection (ICRP) developed the 'as low as reasonably achievable' concept to minimize exposure (5). Although the effects of low-dose exposure have not been clarified, various reports have suggested that exposure is carcinogenic (6-8). As pediatric patients are generally highly radiation-sensitive and have a long life expectancy, it is necessary to pay particular attention to their medical exposure. In 2005, the Japan Radiological Society, Japanese Society of Radiological Technology and Japanese Society of Pediatric Radiology published CT guidelines with the intent to reduce CT exposure dose (9). Therefore, the actual pediatric medical exposure derived from CT scans should be estimated.

The present study aimed to clarify the actual radiation exposure incurred by children during CT examinations and the effective doses in various regions, particularly in 
children aged 0,1 and 5 years, using data collected at Hirosaki University Hospital (Hirosaki, Japan) during a 3-year period from January 2011 to December 2013.

\section{Materials and methods}

Analysis of CT examination. Data of CT examinations performed at Hirosaki University Hospital from January 2011 to December 2013 were extracted from the hospital's radiology information system (RISE-1 Version 1.1.6.0, PSP Corporation, Tokyo, Japan) and analyzed using a Digital Imaging and Communication in Medicine image viewer (EV Insite Version 2.10.6.45, PSP Corporation). The extracted data included the patient's age, examination region, scan protocols, volume CT dose index $\left(\mathrm{CTDI}_{\mathrm{vol}}\right)$ and dose-length product (DLP). Other specific information about each patient was not extracted to avoid a loss of confidentiality. In addition, the original study files were subjected to high security. The files were password-protected for both reading and editing, and only the first author managed the data. Regions on which CT examinations were performed were classified as the head, neck, chest, abdomen, pelvis, coronary and extremity. For example, if one patient had undergone head, abdominal, and pelvic scans, the numbers of patients and regions would be 1 and 3, respectively. Hirosaki University Hospital used the following CT devices for diagnostic radiation: LightSpeed QX/i (GE Healthcare, Waukesha, WI, USA), Discovery CT 750HD (GE Healthcare), SOMATOM Definition (Siemens AG, Erlangen, Germany), SOMATOM Definition AS (Siemens AG) and Aquilion PREMIUM (Toshiba Medical Systems Corp., Otawara, Japan). The present study was approved by the Committee of Medical Ethics of Hirosaki University Graduate School of Medicine (Hirosaki, Japan).

Evaluation of effective doses. Effective doses were calculated using the age-specific and region-specific conversion factors provided in the ICRP Publication 102 (Table I) (10). The effective dose in $\mathrm{mSv}$ was calculated by multiplying the conversion factors by the DLP. Although the present study focused on children aged 0-5 years, Publication 102 only provided conversion factors for children aged 0,1 and 5 years. Therefore, the effective dose evaluation only included children of the following ages: 0 years (up to 11 months after birth), 1 year (up to 1 year and 11 months after birth) and 5 years (up to 5 years and 11 months after birth). The evaluated regions were the head, neck, chest, abdomen and pelvis. Coronary and extremity CT scans were excluded from the evaluation due to a lack of available conversion factors in Publication 102 (10). The clinical scan protocols obtained from the Discovery CT 750HD device, including region, scan type, tube voltage, tube current, noise index, rotation time and pitch are summarized in Table II. The console-displayed CTDI $_{\mathrm{vol}}$ was calculated from the mean tube current when the automatic exposure control (AEC) setting on the Discovery CT 750HD was used. Discovery CT 750HD equipment was used for effective dose estimation in the present study. All displayed DLP data were obtained from the Discovery CT 750HD in accordance with the International Electrotechnical Commission 60601-2-44 Ed.3 (11). At that time, patients without displayed DLP data were excluded from the evaluation, as the effective dose could
Table I. Age-specific and region-specific conversion factors derived from the International Commission on Radiological Protection Publication 102.

\begin{tabular}{lccc}
\hline & \multicolumn{3}{c}{ Patient age, years } \\
\cline { 2 - 4 } Region & 0 & 1 & 5 \\
\hline Head & 0.0110 & 0.0067 & 0.0040 \\
Neck & 0.0170 & 0.0120 & 0.0110 \\
Chest & 0.0390 & 0.0260 & 0.0180 \\
Abdomen & 0.0490 & 0.0300 & 0.0200 \\
Pelvis & 0.0490 & 0.0300 & 0.0200 \\
\hline
\end{tabular}

not be calculated. Exposure doses incurred from positioning images (i.e., scout view) were not evaluated in the present study as they differed in terms of concepts of CTDI ${ }_{\mathrm{vol}}$ and DLP.

To confirm the accuracies of the displayed CTDI ${ }_{\mathrm{vol}}$ and DLP, it was necessary to compare the measured CTDI ${ }_{\mathrm{vol}}$ and DLP. The measured CTDI $_{\text {vol }}$ value of each protocol was measured using a 9015 dosimeter and 10X5-3CT chamber (Radcal Corp., Monrovia, CA, USA). A CTDI phantom (16-cm diameter, IBA Dosimetry GmbH, Schwarzenbruck, Germany) was placed on urethane foam at the CT gantry opening to eliminate an influence of the couch. Each measurement was repeated three times per measurement position (center and four peripheral points) on the Discovery CT 750HD, using scan parameters similar to the clinical protocols (Table II). The CTDI $_{\mathrm{vol}}$ was calculated using corrected temperature and pressure values, a calibration constant, W-value, beam width, gantry rotation number and pitch. The DLP (mGy x cm) was calculated by multiplying the measured $\mathrm{CTDI}_{\mathrm{vol}}(\mathrm{mGy})$ by the scan range $(\mathrm{cm})$. These calculations were based on Publication 102 (10). The scan protocols in the present study were subjected to the $16-\mathrm{cm}$ phantom. As $\sim 50 \%$ of the pediatric radiological examination data were obtained with 750HD and the differences between the measured CTDI ${ }_{\mathrm{vol}}$ and DLP and console-displayed CTDI and DLP had a minimum value of $<2.4 \%$, the following analysis only used examination data obtained with the 750HD.

The advantages of this effective dose estimation method included its user-friendliness and ready availability for estimating pediatric effective $\mathrm{CT}$ doses, particularly in diagnostic facilities without specialized equipment, application tools and knowledge. However, the effective doses obtained with this method did not consider each individual patient, and so the effective doses are a reference value (10), that yield normalized effective doses.

Statistical analysis. Normality tests of the data was performed using the $\chi^{2}$ goodness of fit test to confirm that they were non-parametric data. Significant differences within groups were determined using Kruskal-Wallis tests. The statistical significances of differences among multiple groups were assessed using the Steel-Dwass test for non-parametric multiple comparisons. All statistical analysis were calculated using Excel 2010 Version 14.0.7177.5000 (Microsoft Corp., Redmond, WA, USA) with the Statcel 3 add-on package (OMS 


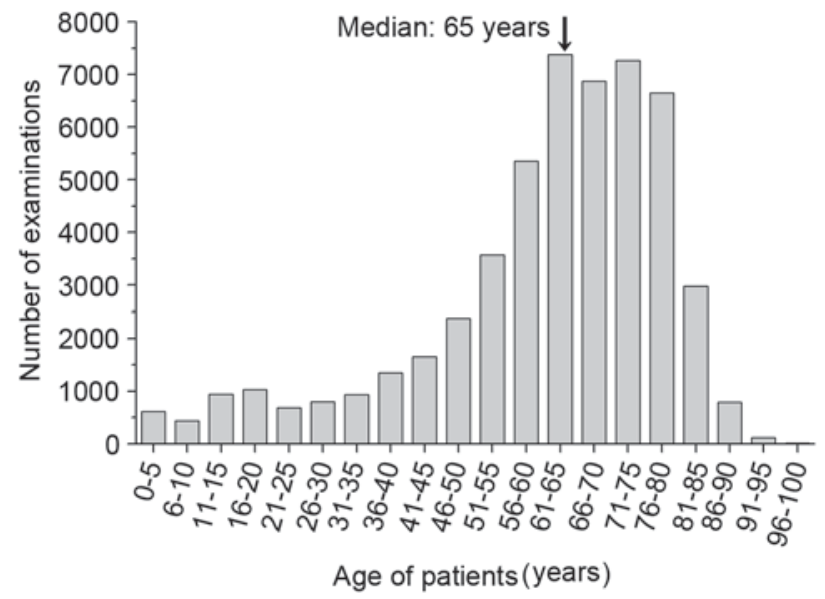

Figure 1. Age distribution of computed tomography examinations at Hirosaki University Hospital (Hirosaki, Japan) during a 3-year period from 2011 to 2013. The median age was 65 years.

publishing Inc., Saitama, Japan). Data were presented as the mean \pm standard deviation. $\mathrm{P}<0.05$ was considered to indicate a statistically significant difference.

\section{Results}

Number of CT examinations. In 2011, 2012 and 2013, 16,662, 17,491 and 17,649 CT examinations were performed, respectively, for a total of 51,802 examinations during the 3-year period. All examinations were classified as follows: Children aged 0-5 years were classified into one group, and all other older subjects were classified into 5 -year categories up to $>96$ years (Fig. 1). The median age was 65 years, and a large proportion of the patients' ages ranged from 61-80 years, with 7,381 and 6,650 cases in the 61-65 and 76-80 year groups, respectively. In total, 613 cases $(1.2 \%)$ involved children aged $0-5$ years. The numbers of $\mathrm{CT}$ examinations per region and age group are summarized in Table III.

Estimated effective doses in children. As previously mentioned, although the present study focused on an analysis of children aged 0-5 years (total 613 cases), conversion factors from Publication 102 were only available for ages 0,1 and 5 years. The present study therefore only evaluated the effective dose for these ages. The effective dose evaluation targeted the 173 cases subjected to diagnostic radiology using the Discovery CT 750HD. The effective doses incurred by children aged 0-5 years are demonstrated in Fig. 2, and the number of examinations are presented in Table IV. The effective dose distribution per examination at each age ranged from 0.66-28.8 mSv, with a mean \pm standard deviation of $5.5 \pm 4.5 \mathrm{mSv}$. Patients with $0.66 \mathrm{mSv}$ of exposure underwent only plain imaging from the neck to chest. In contrast, patients who received an estimated exposure of $28.8 \mathrm{mSv}$ underwent both plain and contrast imaging from the head to pelvis. In the present study, the effective doses at each age ranged from $2.7 \pm 3.0 \mathrm{mSv}$ (5 years group) to $6.3 \pm 4.8 \mathrm{mSv}$ (0 years group). The effective doses received by 5 -year-old patients differed significantly from those of the other age groups $(\mathrm{P}<0.01)$. Subsequently, the effective doses in each region were 
Table III. Number of computed tomography examination regions among children aged 0-5 years.

Region, $\mathrm{n}$

\begin{tabular}{lcrrrrrrrr}
$\begin{array}{l}\text { Age, } \\
\text { years }\end{array}$ & $\begin{array}{c}\text { Number of } \\
\text { patients }\end{array}$ & Head & Neck & Chest & Abdomen & Pelvis & Coronary & Extremity & $\begin{array}{c}\text { Total } \\
\text { regions }\end{array}$ \\
\hline 0 & 221 & 110 & 40 & 108 & 103 & 44 & 0 & 1 & 406 \\
1 & 104 & 55 & 23 & 40 & 44 & 28 & 0 & 0 & 190 \\
2 & 56 & 30 & 8 & 18 & 25 & 15 & 0 & 2 & 98 \\
3 & 73 & 27 & 11 & 25 & 37 & 23 & 0 & 1 & 124 \\
4 & 78 & 39 & 20 & 32 & 30 & 19 & 0 & 0 & 140 \\
5 & 81 & 36 & 24 & 32 & 29 & 17 & 1 & 1 & 140 \\
Total & 613 & 297 & 126 & 255 & 268 & 146 & 1 & 5 & 1,098 \\
\hline
\end{tabular}

Table IV. Number of computed tomography examination regions for effective dose estimation.

\begin{tabular}{lcrrrrrr}
\hline & \multicolumn{5}{c}{ Region, $\mathrm{n}$} \\
\cline { 3 - 6 } Age, years & Number of patients & Head & Neck & Chest & Abdomen & Pelvis & Total regions \\
\hline 0 & 104 & 69 & 20 & 56 & 58 & 26 & 229 \\
1 & 48 & 32 & 8 & 16 & 23 & 17 & 96 \\
5 & 21 & 15 & 5 & 6 & 5 & 5 & 36 \\
Total & 173 & 116 & 33 & 78 & 86 & 48 & 361 \\
\hline
\end{tabular}

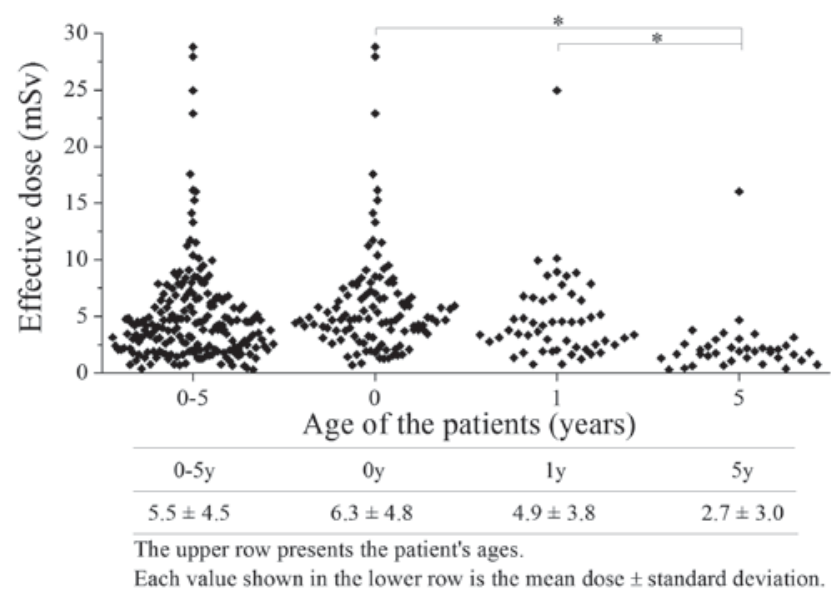

Figure 2. Estimated effective computed tomography examination doses in children aged 0,1 and 5 years. Black dots represent each effective dose. ${ }^{*} \mathrm{P}<0.01$.

compared in each age group. In Fig. 3, the effective dose to the head $(4.3 \pm 2.5 \mathrm{mSv})$ was significantly higher than the doses to the neck $(0.60 \pm 1.3 \mathrm{mSv})$, chest $(1.1 \pm 0.7 \mathrm{mSv})$, abdomen $(2.8 \pm 2.7 \mathrm{mSv})$ and pelvis $(2.0 \pm 1.6 \mathrm{mSv} ; \mathrm{P}<0.01)$. Similarly, the effective dose to the abdomen was significantly higher than those to the neck and chest $(\mathrm{P}<0.01)$. The effective dose to the neck demonstrated the lowest value of all regions evaluated.

The effective doses per region in each age group are demonstrated in Fig. 4. The dose to each of the 5 regions differed according to the age group. In 0- and 1-year-old children, the effective doses to the head $(5.1 \pm 2.4$ and $3.5 \pm 2.6 \mathrm{mSv}$, respectively) were significantly higher than those to the neck of the 0 year group $(0.40 \pm 0.26 \mathrm{mSv} ; \mathrm{P}<0.01)$, chest of the 0 - and 1-year old children $(1.2 \pm 0.71$ and $0.94 \pm 0.54 \mathrm{mSv}$, respectively) and pelvis of the 0 - and 1-year old children $(2.3 \pm 2.0$ and $1.5 \pm 0.88 \mathrm{mSv}$, respectively; $\mathrm{P}<0.01)$. At all ages, the doses to the neck and chest tended to be lower than those of other regions. In the head, chest and pelvis, the conversion factors indicated reduced values with younger ages; a similar trend was observed for the effective doses.

\section{Discussion}

The present study aimed to clarify the actual dose of pediatric radiation exposure incurred during $\mathrm{CT}$ examinations and the effective doses to various regions, particularly in children aged 0,1 and 5 years, using data collected at Hirosaki University Hospital during a 3-year period from January 2011 to December 2013. The proportions of children aged 0-5 years among patients aged 0-15 years who were examined by CT at the Nagasaki University Hospital (Nagasaki, Japan) in 2004 were $\sim 9.5,5.1,4.9,2.9,4.9$ and $4.5 \%$, respectively (12). In the present study, the corresponding proportions were 11.1, 5.2, 2.8, 3.7, 3.9 and 4.1\%, respectively. Although the target age differed, the proportion of CT examinations in the 0 -year group was significantly higher than that in the 1-5 year groups.

The mean effective dose per examination among children aged 0,1 and 5 years was $5.5 \pm 4.5 \mathrm{mSv}$. Furthermore, the estimated effective doses to the head, chest and abdomen were 4.3, 1.1 and $2.8 \mathrm{mSv}$, respectively. A study by Thomas 


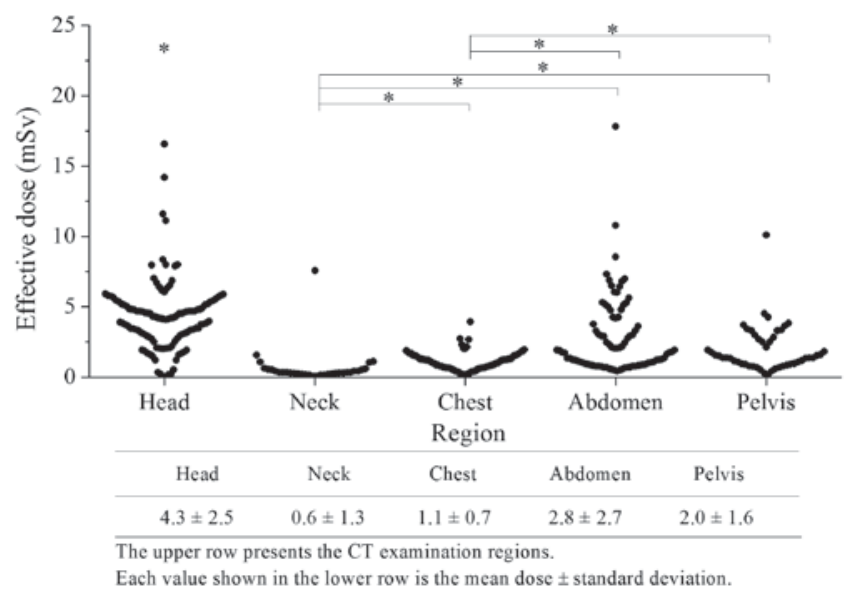

Figure 3. Estimated effective doses according to region. Black dots represent each effective dose. ${ }^{*} \mathrm{P}<0.01$; Head vs. all other regions.

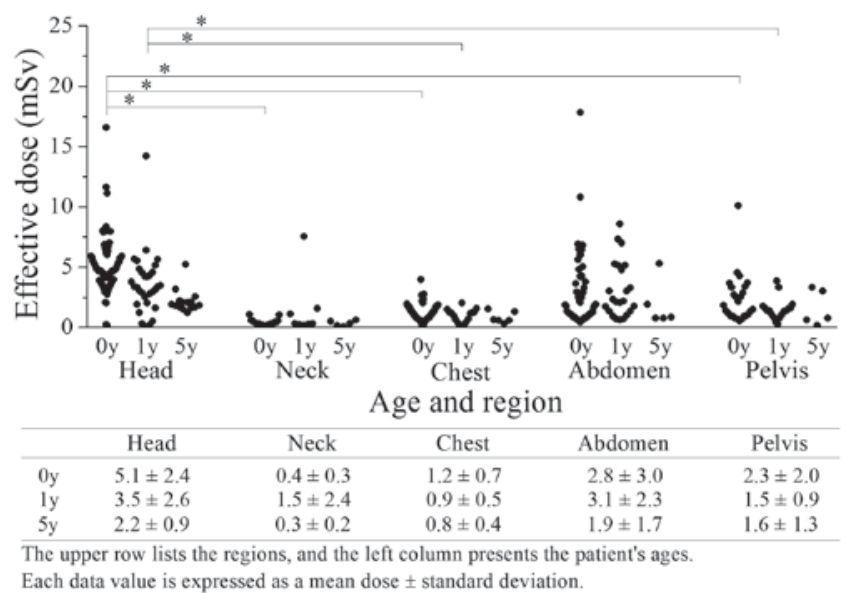

Figure 4. Estimated effective doses according to each age and region. Black dots represent each effective dose. ${ }^{*} \mathrm{P}<0.01$.

and Wang (12) reported that an 0 -year old patient would incur mean effective doses to the head of $4.2 \mathrm{mSv}$, following a single same-region scan in one examination, and $9.1 \mathrm{mSv}$ following two same-region scans in one examination. According to the Japanese pediatric CT guidelines, the effective doses from the reference CT protocol for infants were 3.4 (male) and 3.9 $\mathrm{mSv}$ (female) to the chest, 8.8 (male) and $11.9 \mathrm{mSv}$ (female) to the abdomen (100 kV multiplied by 0.63 ) (9). In children, the effective doses according to the reference CT protocol were 2.1 (male) and $2.5 \mathrm{mSv}$ (female) to the chest, and 7.0 (male) and $8.7 \mathrm{mSv}$ (female) to the abdomen (100 kV multiplied by 0.63) (9). Previously reported doses incurred by other age and region groups were higher than those observed in the present study (12). Therefore, the mean effective doses to all regions in the present study were similar to or lower than the reference and effective doses described in other reports.

Accordingly, in the present study, the results varied widely across all ages and between some regions. Pediatric CT employs a wide range of effective doses, ranging from specific ultralow-dose protocols $(<1 \mathrm{mSv})$ to the extended-coverage body examinations often used for follow-up imaging in oncology and detailed examinations (>810 mSv) (12). A 44-fold difference in the effective dose was observed in the present study. Within each group, the range of effective doses was larger than we had anticipated. Several factors likely contributed to this discrepancy. The first factor encompasses scan methods and ranges. The variation would therefore result from the use of either plain or contrast imaging alone or together, or multiple-phase imaging. Secondly, single-region examinations affected adjacent regions in the cranio-caudal direction, for example, a chest examination may have also included part of the abdomen (liver, spleen, and kidney); therefore, such cases were also counted as abdominal exposure with respect to effective dose and number. In the present study, even scans with small ranges were classified according to region and were included in the calculations. Some variability in scan length may be expected due to of operator variability, and body examinations vary according to patient height. Such variability was most often encountered in head CT scans, where selected axial images were occasionally repeated if patient motion had significantly degraded the image quality. Also, for head and neck scans, neck images were obtained using the same scan parameters as the head. As a result, large variation occurred in these cases. Furthermore, each age group may include considerable ranges in patient height and weight. Tube current with AEC was determined by calculating the body thickness and size from positioning images. The present study elected to include these patients in the study as the purpose was to reflect actual situations and effective doses.

The displayed DLP conversion factor-based methodology used in the present study demonstrated some limitations and issues. According to a study by Kobayashi et al (13), effective doses estimated using conversion factors differed from the measured doses by $20 \%$, even in the simplest scans. Patients who underwent coronary artery and extremity CT evaluations were excluded from the present study as conversion factors have not been reported for these regions (14). Although AEC is applied to optimize the dose to the body size, the displayed $\mathrm{CTDI}_{\mathrm{vol}}$ would cause uncertainty by averaging the tube current value. Therefore, it is difficult to indicate an assessed dose that is suitable for a particular body size using the effective doses in the present study, as these effective doses are reference value, specifically normalized effective doses (10).

Despite these limitations, the simple approach used in the present study to estimate the radiation exposure from CT examinations is adequate for current data and technology $(13,15)$. The effectiveness of diagnostic imaging is widely and generally known, and the number of CT scanners may be used as an index of hospital quality (1). Compared with other countries, Japanese patients expect higher levels of medical care and seek treatment at hospitals owning CT scanners (1). The strength of the displayed DLP conversion factor-based method is readily available to all radiologists, clinicians and technologists. Furthermore, this method may be used to contribute to pediatric CT examination and diagnostic reference level surveys.

In Japan, no official system is responsible for the quality control of CT examinations and patient radiation exposure (4). In addition, it is difficult to accurately quantify the CT risk level that would require long-term follow-up of a majority of patients $(16,17)$. Reports concerning risks and CT doses have indicated a low level of awareness and knowledge among 
medical staff $(18,19)$. The present study may allow medical staff to understand and recognize the significance of a wide effective dose range that encompasses specific low-dose protocols to extended-coverage body scans. In addition, this effective dose estimation method may serve as an important reference value that allows specific values for effective doses to be obtained, and includes the advantage of being a user-friendly and rapidly available method for the estimation of pediatric CT effective doses, particularly in diagnostic facilities without specialized equipment, application tools, and/or knowledge. In conclusion, it is necessary to consider the requirement for a prospective exposure management system in each patient, as well as the estimation of each patient's low-dose radiation exposure $(20,21)$. The introduction of a cancer registration according to medical exposure and a resulting medical exposure dose management system would leave a great legacy for future generations.

\section{Acknowledgements}

The present study was supported by Hirosaki University Institutional Research (2012, IK).

\section{References}

1. Katada K: CT radiation exposure in Japan: Analysis of social factors. Nihon Hoshasen Gijutsu Gakkai Zasshi 62: 649-656, 2006 (In Japanese).

2. The silver market phenomenon. Business opportunities in an Era of demographic change. Kohlbacher F and Herstatt C (eds). Springer, Germany, pp427-441, 2008.

3. Yoshioka M, Shirayama Y, Imoto I, Hinode D, Yanagisawa S and Takeuchi Y: Current status of collaborative relationships between dialysis facilities and dental facilities in Japan: Results of a nationwide survey. BMC Nephrol 16: 17, 2015.

4. Tsushima Y, Taketomi-Takahashi A, Takei H, Otake $\mathrm{H}$ and Endo K: Radiation exposure from CT examinations in Japan. BMC Med Imaging 10: 24, 2010.

5. Implications of Commission recommendations that doses be kept as low as readily achievable. A report of ICRP Committee 4 1CRP Publication 22. Pergamon Press, Oxford, pp2-3, 1977.

6. Brenner D, Elliston C, Hall E and Berdon W: Estimated risks of radiation-induced fatal cancer from pediatric CT. AJR Am J Roentgenol 176: 289-296, 2001.

7. Krille L, Zeeb H, Jahnen A, Mildenberger P, Seidenbusch M, Schneider K, Weisser G, Hammer G, Scholz P and Blettner M: Computed tomographies and cancer risk in children: A literature overview of CT practices, risk estimations and an epidemiologic cohort study proposal. Radiat Environ Biophys 51: 103-111, 2012.
8. Mathews JD, Forsythe AV, Brady Z, Butler MW, Goergen SK, Byrnes GB, Giles GG, Wallace AB, Anderson PR, Guiver TA, et al: Cancer risk in 680,000 people exposed to computed tomography scans in childhood or adolescence: Data linkage study of 11 million Australians. BMJ 346: f2360, 2013.

9. Japanese Radiological Society: Management of patient dose in pediatric computed tomography. Nihon Hoshasen Gijutsu Gakkai Zasshi 61: 493-495, 2005 (In Japanese).

10. Valentin J; International Commission on Radiation Protection: Managing patient dose in multi-detector computed tomography(MDCT). ICRP Publication 102. Ann ICRP 37: 1-79, iii, 2007.

11. International Electrotechnical Commission: Medical electrical equipment-Part 2-44: Particular requirements for the basic safety and essential performance of X-ray equipment for computed tomography. 3rd edition. IEC International Standard 60601-2-44: IEC, Geneva, 2009.

12. Thomas KE and Wang B: Age-specific effective doses for pediatric MSCT examinations at a large children's hospital using DLP conversion coefficients: A simple estimation method. Pediatr Radiol 38: 645-656, 2008.

13. Kobayashi M, Ootsuka T and Suzuki S: Evaluation and examination of accuracy for the conversion factors of effective dose per dose-length product. Nihon Hoshasen Gijutsu Gakkai Zasshi 69: 19-27, 2013 (In Japanese).

14. Ghotbi N, Ohtsuru A, Ogawa Y, Morishita M, Norimatsu N, Namba H, Moriuchi H, Uetani M and Yamashita S: Pediatric CT scan usage in Japan: Results of a hospital survey. Radiat Med 24: 560-567, 2006

15. Brady Z, Cain TM and Johnston PN: Justifying referrals for paediatric CT. Med J Aust 197: 95-99, 2012.

16. Berrington de González A, Mahesh M, Kim KP, Bhargavan M, Lewis R, Mettler F and Land C: Projected cancer risks from computed tomographic scans performed in the United States in 2007. Arch Intern Med 169: 2071-2077, 2009.

17. Preston RJ, Boice JD Jr, Brill AB, Chakraborty R, Conolly R, Hoffman FO, Hornung RW, Kocher DC, Land CE, Shore RE and Woloschak GE: Uncertainti in estimating health risks associated with exposure to ionising radiation. J Radiol Prot 33: 573-588, 2013.

18. Merzenich H, Krille L, Hammer G, Kaiser M, Yamashita S and Zeeb H: Paediatric CT scan usage and referrals of children to computed tomography in Germany-a cross-sectional survey of medical practice and awareness of radiation related health risks among physicians. BMC Health Serv Res 12: 47, 2012.

19. Brown $\mathrm{N}$ and Jones L: Knowledge of medical imaging radiation dose and risk among doctors. J Med Imaging Radiat Oncol 57: 8-14, 2013.

20. Eisenberg MJ, Afilalo J, Lawler PR, Abrahamowicz M, Richard H and Pilote L: Cancer risk related to low-dose ionizing radiation from cardiac imaging in patients after acute myocardial infarction. CMAJ 183: 430-436, 2011.

21. Hiromitsu O: Health risk assessment of exposure to ionizing radiation. J Natl-Inst-Public Health 60: 326-631, 2011. 